\title{
Serotype/serogroup-specific antibiotic non-susceptibility of invasive and non-invasive Streptococcus pneumoniae, Switzerland, 2004 to 2014
}

\author{
C Hauser ${ }^{12}$, A Kronenberg ${ }^{12}$, A Allemann ${ }^{13}$, K Mühlemann ${ }^{124}$, M Hilty ${ }^{12}$ \\ 1. Institute for Infectious Diseases, Faculty of Medicine, University of Bern, Bern, Switzerland \\ 2. Department of Infectious Diseases, Bern University Hospital, University of Bern, Bern, Switzerland \\ 3. Graduate School for Cellular and Biomedical Sciences, University of Bern, Bern, Switzerland \\ 4. Deceased
}

Correspondence: Markus Hilty (Markus.Hilty@ifik.unibe.ch)

Hauser C, Kronenberg A, Allemann A, Mühlemann K, Hilty M. Serotype/serogroup-specific antibiotic non-susceptibility of invasive and non-invasive Streptococcus pneumoniae, Switzerland, 2004 to 2014. Euro Surveill. 2016;21(21):pii=30239. DOI: http://dx.doi.org/10.2807/1560-7917.ES.2016.21.21.30239

Concurrent analysis of antibiotic resistance of colonising and invasive Streptococcus pneumoniae gives a more accurate picture than looking at either of them separately. Therefore, we analysed 2,129 non-invasive and 10,996 invasive pneumococcal isolates from Switzerland from 2004 to 2014 , which spans the time before and after the introduction of the heptavalent $\left(\mathrm{PCV}_{7}\right)$ and 13-valent (PCV13) conjugated pneumococcal polysaccharide vaccines. Serotype/serogroup information was linked with all antibiotic resistance profiles. During the study period, the proportion of non-susceptible non-invasive and invasive isolates significantly decreased for penicillin, ceftriaxone, erythromycin and trimethoprim/sulfamethoxazole (TMP-SMX). This was most apparent in non-invasive isolates from study subjects younger than five years (penicillin $(p=0.006)$, erythromycin $(p=0.01)$ and TMP-SMX $(p=0.002))$. Resistant serotypes/serogroups included in $\mathrm{PCV}_{7}$ and/or $\mathrm{PCV}_{13}$ decreased and were replaced by non-PCV13 serotypes (6C and 15B/C). Serotype/serogroup-specific antibiotic resistance rates were comparable between invasive and non-invasive isolates. Adjusted odds ratios of serotype/serogroup-specific penicillin resistance were significantly higher in the west of Switzerland for serotype 6B $(1.8 ; 95 \%$ confidence interval (Cl): 1.4-4.8), 9V (3.4; 95\% Cl: 2.0-5.7), 14 (5.3; 95\% Cl: 3.8-7.5), 19A (2.2; 95\% Cl: $1.6-3.1)$ and $19 \mathrm{~F}(3.1 ; 95 \% \mathrm{Cl}: 2.1-4.6)$, probably due to variations in the antibiotic consumption.

\section{Introduction}

Antibiotic resistance in Streptococcus pneumoniae is a worldwide concern and can lead to treatment failures with increase in morbidity and mortality, augmented treatment cost and use of more toxic reserve antimicrobials $[1,2]$. In this context, surveillance of antibiotic resistance in S. pneumoniae is important for the appropriate choice of empirical therapy, to detect new resistance developments in a timely manner and to monitor the effect of interventions such as antibiotic stewardship campaigns or vaccines on resistance rates and serotype distribution [3].

Heptavalent conjugated pneumococcal polysaccharide vaccine $\left(P C V_{7}\right)$ followed by 13 -valent vaccine (PCV13) have been recommended and reimbursed by the health insurance in Switzerland since late 2006 and 2011, respectively, for all children younger than two years [4]. During the period from 2008 to 2010, the pneumococcal vaccine coverage was, respectively, ca 50\% (95\% confidence interval (Cl): 46.0-53.5) and $37 \%$ (95\% Cl: 32.5-41.6) for two and three doses at the age of two years [4]. The coverage increased to $79 \%$ (95\% $\mathrm{Cl}: 77.4-80.4)$ and $75 \%(95 \% \mathrm{Cl}: 73.8-76.7)$, respectively, for two and three doses between 2011 and 2013. Introduction of vaccines led to a decrease in the incidence in invasive pneumococcal disease (IPD) but also to a change in serotype redistribution across all ages [4]. In other countries, vaccine introduction has additionally led to a decrease in antibiotic resistance rates because the less susceptible serotypes have been included in the vaccines [5]. So far, in Switzerland, antibiotic resistance in $S$. pneumoniae has only been analysed in the pre-PCV7 era from 2001 to 2004 [6].

Ideally, antibiotic resistance rates in non-invasive and invasive $S$. pneumoniae are analysed simultaneously, but such studies of representative size are rare or often not very recent $[6,7]$ because in many countries, antibiotic resistance data is exclusively drawn from surveillance of invasive isolates. Switzerland runs two different national surveillance systems collecting resistance data on S. pneumoniae: sentinel surveillance 


\section{A. IPD $<5$ years}

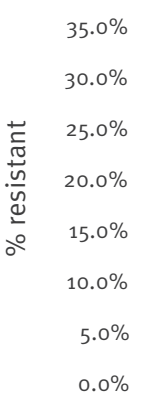

$.0 \%$

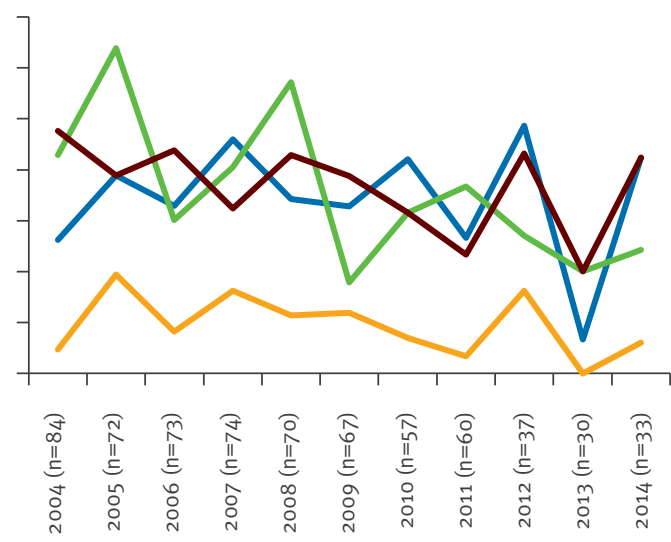

Year

\section{IPD $>64$ years}

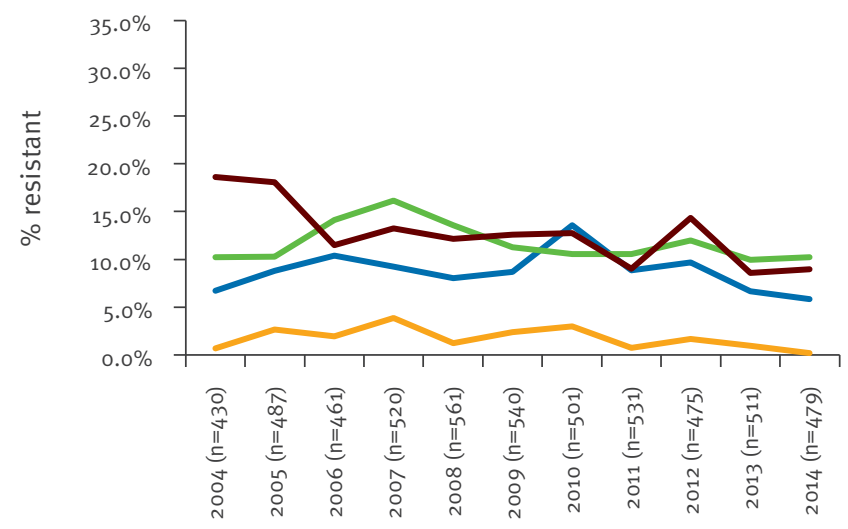

Year
B. IPD 5-64 years

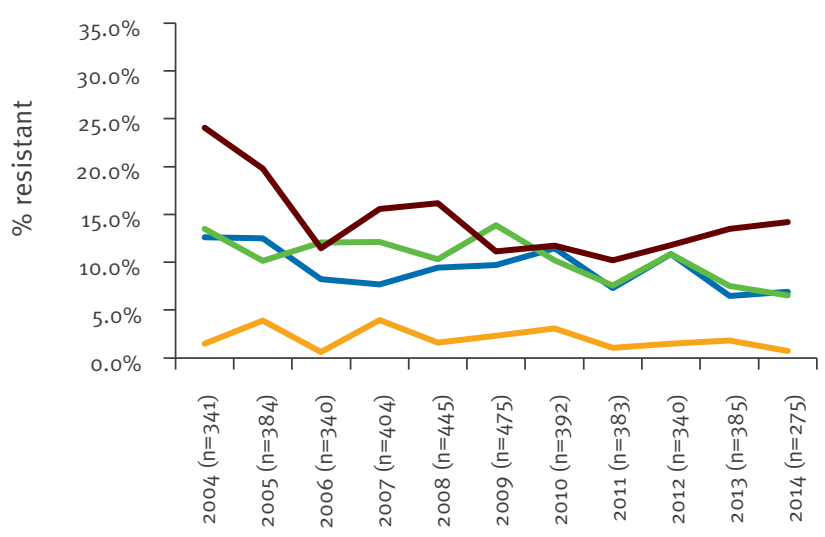

Year

D. Non-IPD $<5$ years

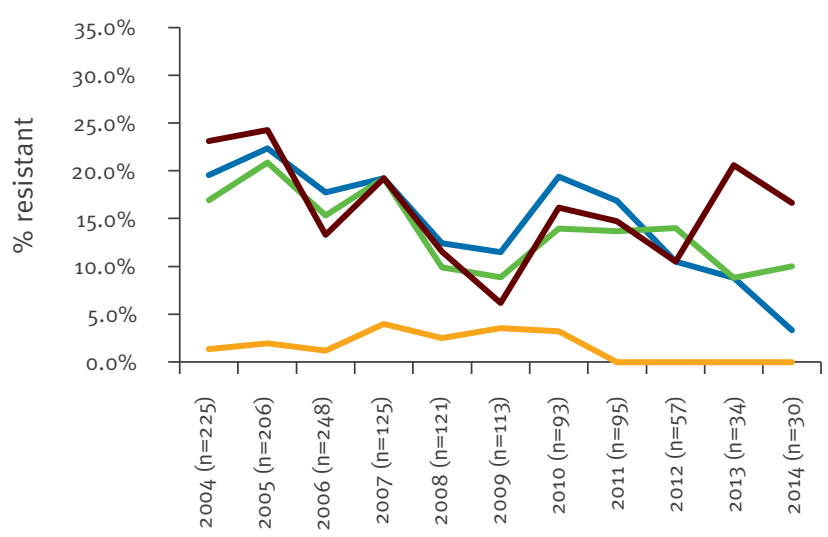

Year

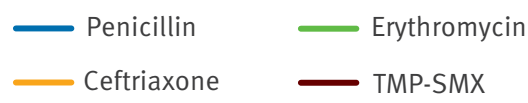

IPD: invasive pneumococcal disease; TMP-SMX: trimethoprim/sulfamethoxazole.

Antibiotic resistance towards penicillin includes isolates with MIC > $0.06 \mathrm{mg} / \mathrm{L}$

Samples for which age group was not known $(n=681)$ are excluded from this analysis.

Analyses were stratified according to IPD in patients $<5$ years (A), $5-64$ years (B), $>64$ years of age (C) and non-IPD in patients $<5$ years of age (D). Data on non-IPD in patients 5-64 years and $>64$ years of age were omitted due to low number of isolates in these two categories. 
of outpatient non-invasive pneumococci (Sentinella) and comprehensive passive surveillance of all invasive pneumococci $[4,8]$. The aims of this study were (i) to simultaneously describe the prevalence of antibiotic resistance in invasive and non-invasive S. pneumoniae in different patient populations in Switzerland from 2004 to 2014, (ii) to analyse possible temporal trends and effects of $\mathrm{PCV}_{7}$ and $\mathrm{PCV}_{13}$ on resistance prevalence, (iii) to detect serotype/serogroup-specific antibiotic resistance and (iv) to analyse regional differences for the antibiotic resistance rates.

\section{Methods}

Sentinel surveillance of non-invasive pneumococcal isolates (Sentinella)

Between 2004 and 2014, data on colonising pneumococci were obtained from a nationwide, ongoing, prospective surveillance study within the Swiss Sentinel System which has been described in detail previously [8]. In brief, this network involves a chosen sample of practitioners who represent Switzerland geographically and demographically. The overall number of participants per subspecialty in the Sentinel System is defined as a proportion of all Swiss practitioners in the matching specialty $[8,9]$. Therefore, ca 200 practitioners (general practitioners, internists and paediatricians) took samples of outpatients who were clinically diagnosed with acute otitis media or pneumonia [8]. All received swabs were cultured for S. pneumoniae at the Swiss National Reference Centre for Pneumococci (NZPn) as described [6].

\section{Comprehensive surveillance of invasive pneumococcal isolates}

Physician reporting of invasive pneumococcal infection has been mandatory in Switzerland since 1999 (http:// www.bag.admin.ch). In March 2002, the NZPn was set up and has since been prospectively collecting clinical pneumococcal isolates from normally sterile body sites (blood, cerebrospinal, joint, pleural and peritoneal fluid but not middle ear fluid) sent in by Swiss clinical microbiology laboratories. It is possible to link ca $90 \%$ of physician-reported IPD cases with a corresponding pneumococcal isolate, indicating a very high participation of involved laboratories and a high completeness of data linkage [4]. This study uses only the NPZn isolates and demographic data associated with them. Physician-reported data are not included in the analysis in this study.

\section{Analysis of non-invasive and invasive isolates}

All isolates were confirmed as S. pneumoniae by alpha haemolysis morphology on blood agar plates, bile solubility and optochin sensitivity. Serotypes of all confirmed pneumococcal isolates were determined by the Quellung reaction.

Methods for antibiotic resistance testing were identical for non-invasive and invasive isolates and have been described previously [8]. In brief, all isolates were tested against oxacillin ( $1 \mu \mathrm{g}$ disk), erythromycin, trimethoprim/sulfamethoxazole (TMP-SMX) and levofloxacin by the disk diffusion method. For isolates with reduced susceptibility to oxacillin, the minimum inhibitory concentration (MIC) against penicillin and ceftriaxone was determined by Etest (AB Biodisk) according to Clinical and Laboratory Standards Institute (CLSI) [10]. During the study period, CLSI introduced new MIC breakpoints for parenteral penicillin therapy of nonmeningitis infections. To ensure that the same breakpoints were applied for all isolates over the entire study period, we used the oral non-meningitis breakpoints (susceptible $(\mathrm{S}) \leq 0.06 \mathrm{mg} / \mathrm{L}$ or oxacillin disk diameter $\geq 20 \mathrm{~mm}$ ) for penicillin non-susceptibility and the meningitis $(S \leq 0.5 \mathrm{mg} / \mathrm{L})$ breakpoints for ceftriaxone non-susceptibility for the entire time period.

For this study, data for isolates collected between January 2004 and December 2014 were analysed. Data on patients' age, sex and geographical origin of samples were available for all isolates except 681 records with missing data on age and 6 records with missing data on geographical region.

\section{Statistical analysis}

Resistance prevalence data were stratified by age group ( $(5,5-64$ and $>64$ years), serotype/serogroup and geographical region. For geographical comparisons, Switzerland was divided into two regions, the French speaking western part designated 'west' and the remaining parts of the country including the Italian speaking canton Ticino designated 'other', as described previously [6]. Differences were calculated using $2 \times 2$ or $3 \times 2$ chi-square test. Changes over time from 2004 to 2014 were analysed with the chi-square test for trend with CDC Epilnfo Version 7 and GraphPad Prism version 6.00 for Windows, GraphPad Software. A cut-off value of $p \leq 0.05$ (two-tailed) was used for these tests.

Serotype/serogroup-specific antibiotic non-susceptibilities of pencillin, erythormycin or TMP-SMX of invasive and colonising $S$. pneumoniae isolates were calculated by multivariate logistic regression analysis. Adjusted odds ratios (aOR) with $95 \%$ confidence intervals $(95 \% \mathrm{Cl})$ were received for serotypes/serogroups with overall proportions of above $1 \%$. For all six regression analyses, the remaining serotypes, consisting of serotypes/serogroups with overall proportions below $1 \%$ in invasive and non-invasive S. pneumoniae, served as the reference group.

Serotype/serogroup-specific antibiotic resistance by geographical region and isolation site (i.e. colonising vs invasive $S$. pneumoniae) for the top four resistant serotypes for penicillin, erythromycin and trimethoprim/sulfamethoxazole (TMP-SMX) was also calculated by multivariate logistic regression analysis. Isolates from the 'other' part of Switzerland were used as the reference group for each of the 12 regression analyses. 
FIGURE 2

Serotype/serogroup-specific multivariate logistic regression analysis, Streptococcus pneumoniae isolates, Switzerland, $2004-14(\mathrm{n}=12,438)$

A. Non-IPD penicillin

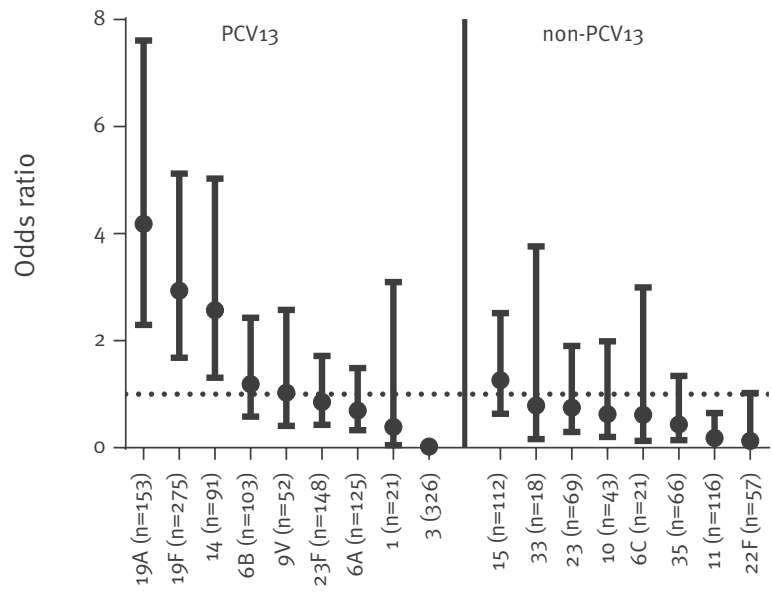

Serotypes/serogroups

C. Non-IPD TMP-SMX

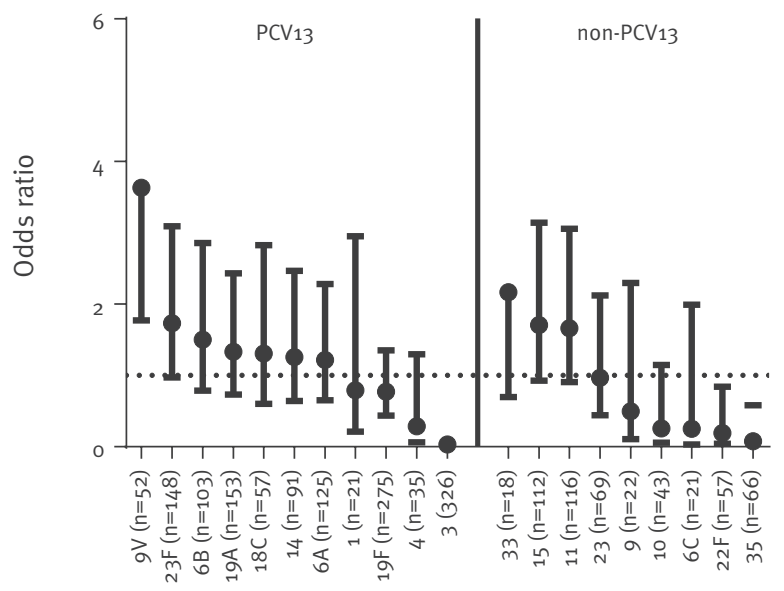

Serotypes/serogroups

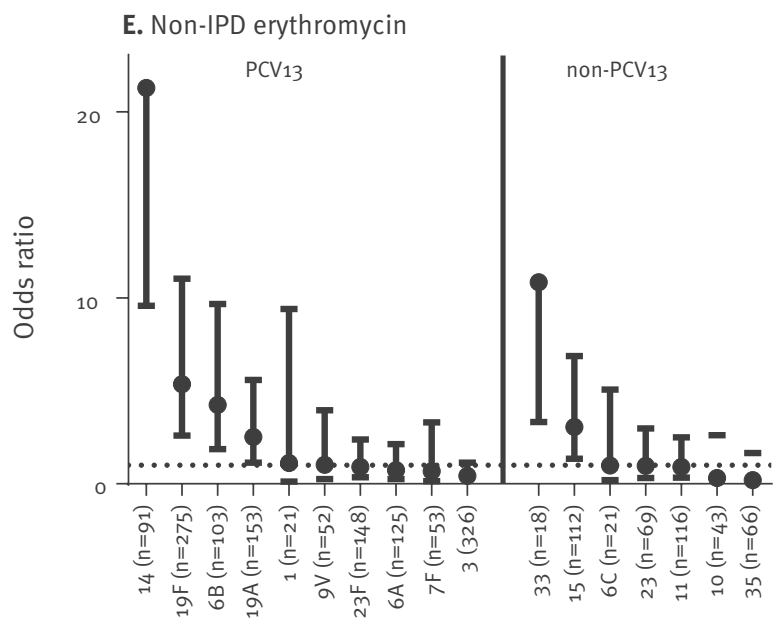

B. IPD penicillin

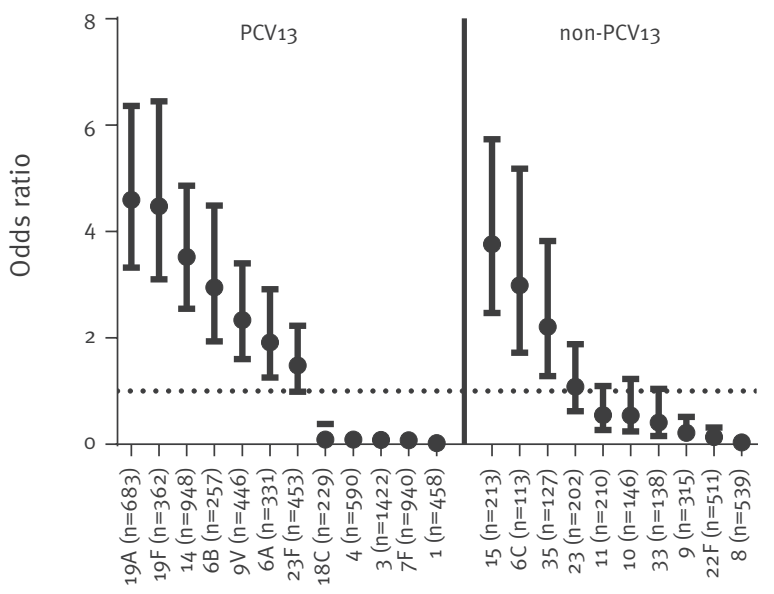

Serotypes/serogroups

D. ID TMP-SMX

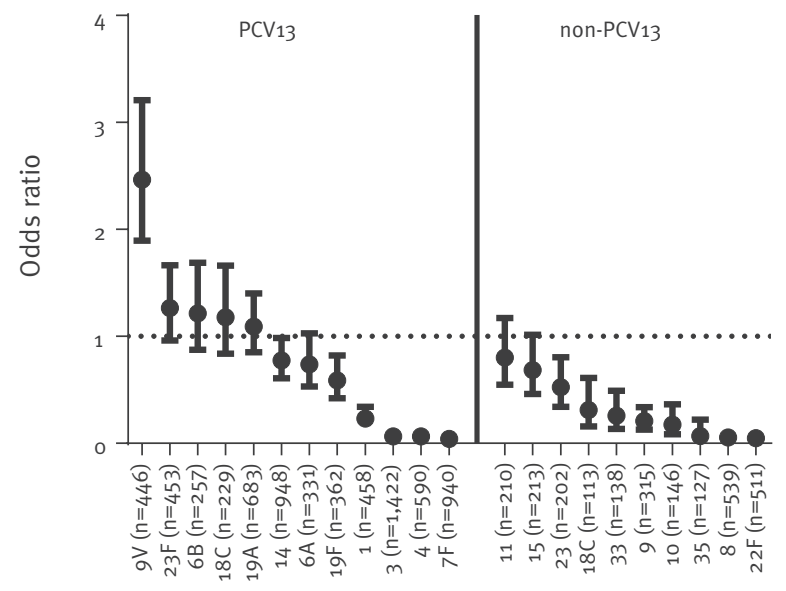

Serotypes/serogroups

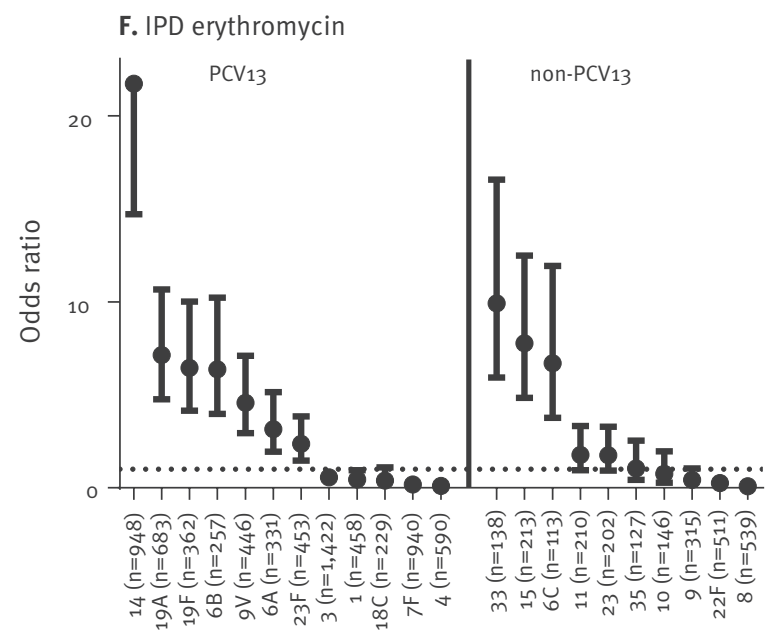

Serotypes/serogroups

Serotypes/serogroups

IPD: invasive pneumococcal disease; PCV: pneumococcal conjugated vaccine; TMP-SMX: trimethoprim/sulfamethoxazole.

Analysis of serotype/serogroups with penicillin non-susceptibility in non-invasive (A) and invasive (B) samples. TMP-SMX non-susceptibility is illustrated for non-invasive (C) and invasive (D) samples. Erythromycin non-susceptibility is shown for non-invasive (E) and invasive (F) samples. Odds ratios were adjusted for year of isolation, geographical region (west vs other) and age category ( $\langle 5 ; 5-64$ and $>64$ years). Serotypes/serogroups with proportion of $<1 \%$ were combined to one group for the analysis and served as reference group. In the reference group, there were 680 invasive and 148 colonising isolates, respectively. PCV 13 serotypes are indicated. PCV 7 serotypes are included in the PC V13 serotypes and are $4,6 \mathrm{~B}, 9 \mathrm{~V}, 14,18 \mathrm{C}, 19 \mathrm{~F}$ and $23 \mathrm{~F}$.

Isolates with unknown age $(n=681)$ and/or geographical region known $(n=6)$ were excluded.

4

www.eurosurveillance.org

Check for updates 


\begin{tabular}{|c|c|c|c|c|c|}
\hline & \multicolumn{2}{|c|}{ Non-invasive Streptococcus pneumoniae } & \multicolumn{2}{|c|}{ Invasive Streptococcus pneumoniae } & \multirow[b]{2}{*}{$p^{a}$} \\
\hline & $\mathrm{n}$ & $\%$ & $n$ & $\%$ & \\
\hline Total isolates & 2,129 & 100.0 & 10,996 & 100.0 & NA \\
\hline \multicolumn{6}{|l|}{ Age } \\
\hline$<5$ years & 1,347 & 63.3 & 657 & 6.0 & $<0.001$ \\
\hline $5-64$ years & 710 & 33.3 & 4,164 & 37.9 & $<0.001$ \\
\hline$>64$ years & 70 & 3.3 & 5,496 & 50.0 & $<0.001$ \\
\hline Not known & 2 & 0.1 & 679 & 6.2 & $<0.001$ \\
\hline \multicolumn{6}{|l|}{ Region } \\
\hline West & 856 & 40.2 & 2,781 & $25 \cdot 3$ & $<0.001$ \\
\hline Other & 1,271 & 59.7 & 8,211 & 74.7 & $<0.001$ \\
\hline Not known & 2 & 0.1 & 4 & 0.0 & 0.3 \\
\hline \multicolumn{6}{|l|}{ Non-susceptibility } \\
\hline Penicillin ${ }^{b}$ & 299 & 14.0 & 1,077 & 9.8 & $<0.0001$ \\
\hline Ceftriaxone & 35 & 1.6 & 242 & 2.2 & 0.04 \\
\hline Erythromycin & 256 & 12.0 & 1,295 & 11.8 & 0.7 \\
\hline TMP-SMX & 328 & 15.4 & 1,540 & 14.0 & 0.09 \\
\hline Levofloxacin & 1 & 0.0 & 41 & 0.4 & 0.02 \\
\hline \multicolumn{6}{|l|}{ Serotype/serogroup ${ }^{c}$} \\
\hline Age $<5$ years & \multicolumn{2}{|c|}{$\mathrm{n}=1,347$} & \multicolumn{2}{|c|}{$n=657$} & \\
\hline $\mathrm{PCV}_{7}$ & 532 & 39.5 & 242 & 36.8 & 0.3 \\
\hline $\mathrm{PCV}_{13}$ minus $\mathrm{PCV}_{7}$ & 379 & 28.1 & 280 & 42.6 & $<0.0001$ \\
\hline Non-PCV13 & 436 & 32.4 & 135 & 20.4 & $<0.0001$ \\
\hline Age 5-64 years & \multicolumn{2}{|c|}{$\mathrm{n}=710$} & \multicolumn{2}{|c|}{$n=4,164$} & \\
\hline $\mathrm{PCV}_{7}$ & 212 & 29.9 & 1,228 & 29.5 & 0.8 \\
\hline $\mathrm{PCV}_{13}$ minus $\mathrm{PCV}_{7}$ & 283 & 39.9 & 1,667 & 40.0 & 0.9 \\
\hline Non-PCV13 & 215 & 30.3 & 1,269 & 30.5 & 0.9 \\
\hline Age $>64$ years & \multicolumn{2}{|c|}{$n=70$} & \multicolumn{2}{|c|}{$n=5,495$} & \\
\hline $\mathrm{PCV}_{7}$ & 17 & 24.3 & 1,816 & 33.0 & 0.1 \\
\hline $\mathrm{PCV}_{13}$ minus $\mathrm{PCV}_{7}$ & 18 & 25.7 & 1,911 & 34.8 & 0.1 \\
\hline Non-PCV13 & 35 & 50.0 & 1,768 & 31.2 & 0.002 \\
\hline
\end{tabular}

NA: not applicable; TMP-SMX: trimethoprim/sulfamethoxazole.

a $p$ value calculated with $2 \times 2$ chi-square test.

${ }^{\mathrm{b}}$ Minimal inhibitor concentration $>0.06 \mathrm{mg} / \mathrm{L}$.

'PCV7: serotypes/serogroups 4, 6B, 9V, 14, 18C, 19F, 23F; PCV13 minus PCV7: serotypes/serogroups 1, 3, 5, 6A, 7F, 19A; PCV13: serotypes/ serogroups $4,6 \mathrm{~B}, 9 \mathrm{~V}, 14,18 \mathrm{C}, 19 \mathrm{~F}, 23 \mathrm{~F}, 1,3,5,6 \mathrm{~A}, 7 \mathrm{~F}, 19 \mathrm{~A}$; Non-PCV13: all remaining serotypes/serogroups. For two and 679 non-invasive and invasive isolates, respectively, age was unknown. For one invasive isolate, the serotype/serogroup was unknown.

Logistic regression analyses were performed using Stata (version 13.0).

\section{Results}

In total, 2,129 non-invasive and 10,996 invasive pneumococcal isolates from January 2004 to December 2014 were analysed (Table 1). The patient population with non-invasive $S$. pneumoniae had a significantly higher proportion of children younger than five years than the patient population with invasive isolates (Table $1 ; p<0.001$ ). This is because non-invasive isolates were received from patients with pneumonia $(n=285)$, but more often from patients with acute otitis media $(n=1,775)$ who are mostly toddlers. For 69 isolates, the underlying disease was unknown. In general, isolates from the west were overrepresented among the non-invasive isolates and proportional to the resident population among the invasive isolates (Table 1 ).

Overall, we identified 22 serotypes/serogroups with a prevalence of more than $1.0 \%$ (serotypes/serogroups 1 , $3,4,6 \mathrm{~A}, 6 \mathrm{~B}, 6 \mathrm{C}, 7 \mathrm{~F}, 8,9,9 \mathrm{~V}, 10,11,14,15,18 \mathrm{C}, 19 \mathrm{~A}$, $19 \mathrm{~F}, 22 \mathrm{~F}, 23,23 \mathrm{~F}, 33$ and 35 ). The proportions of isolates with $\mathrm{PCV}_{7}$ serotypes did not differ between the non-invasive and the invasive isolates in any of the three chosen age categories ( 15 years, 5-64 years and $>64$ years). In contrast, differences were noted for the $\mathrm{PCV}_{13}$ minus $\mathrm{PCV}_{7}$ (serotypes/serogroups 1, 3, $5,6 \mathrm{~A}, 7 \mathrm{~F}, 19 \mathrm{~A})$ and non-PCV13 serotypes/serogroups 
TABLE 2

Antibiotic-resistant pneumococcal isolates by age and geographical origin of samples, Switzerland, 2004-14 (n = 13,125)

\begin{tabular}{|c|c|c|c|c|c|c|c|c|c|c|c|c|}
\hline & \multicolumn{2}{|c|}{$\langle 5$ years } & \multicolumn{2}{|c|}{$5-64$ years } & \multicolumn{2}{|c|}{$>64$ years } & \multirow{2}{*}{$p^{a}$} & \multicolumn{2}{|c|}{ West $^{\mathrm{b}}$} & \multicolumn{2}{|c|}{ Other ${ }^{c}$} & \multirow{2}{*}{$P^{d}$} \\
\hline & $\mathrm{n}$ & $\%$ & $\mathrm{n}$ & $\%$ & $n$ & $\%$ & & $\mathrm{n}$ & $\%$ & $n$ & $\%$ & \\
\hline \multicolumn{13}{|l|}{ Resistance Invasive } \\
\hline $\begin{array}{l}\text { Penicillin } \\
\text { (MIC > } 0.06 \mathrm{mg} / \mathrm{L} \text { ) }\end{array}$ & 114 & 17.4 & 392 & 9.4 & 483 & 8.8 & $<0.0001$ & 449 & 16.1 & 628 & 7.6 & $<0.0001$ \\
\hline $\begin{array}{l}\text { Penicillin } \\
(\text { MIC > } 2.0 \mathrm{mg} / \mathrm{L})\end{array}$ & 10 & 1.5 & 32 & 0.8 & 28 & 0.5 & 0.008 & 30 & 1.1 & 45 & 0.5 & 0.003 \\
\hline Ceftriaxone $^{\mathrm{e}}$ & 33 & 5.0 & 86 & 2.1 & 98 & 1.8 & $<0.0001$ & 121 & 4.4 & 121 & 1.5 & $<0.0001$ \\
\hline Erythromycine & 125 & 19.0 & 440 & 10.6 & 646 & 11.8 & $<0.0001$ & 425 & 15.3 & 870 & 10.6 & $<0.0001$ \\
\hline TMP-SMXe & 124 & 18.9 & 601 & 14.4 & 693 & 12.6 & $<0.0001$ & 479 & 17.2 & 1059 & 12.9 & $<0.0001$ \\
\hline Levofloxacin $^{\mathrm{e}}$ & 1 & 0.2 & 8 & 0.2 & 29 & 0.5 & 0.02 & 10 & 0.4 & 31 & 0.4 & 0.9 \\
\hline Totalf & 657 & 100.0 & 4,164 & 100.0 & 5,496 & 100.0 & NA & 2,781 & 100.0 & 8,211 & 100.0 & NA \\
\hline \multicolumn{13}{|c|}{ Resistance non-invasive } \\
\hline $\begin{array}{l}\text { Penicillin } \\
\text { (MIC > } 0.06 \mathrm{mg} / \mathrm{L} \text { ) }\end{array}$ & 226 & 16.8 & 70 & 9.9 & 3 & $4 \cdot 3$ & $<0.0001$ & 175 & 20.4 & 124 & 9.8 & $<0.0001$ \\
\hline $\begin{array}{l}\text { Penicillin } \\
(\text { MIC > } 2.0 \mathrm{mg} / \mathrm{L})\end{array}$ & 9 & 0.7 & 3 & 0.4 & 1 & 1.4 & 0.5 & 9 & 1.1 & 4 & 0.3 & 0.03 \\
\hline Ceftriaxone $^{e}$ & 25 & 1.9 & 9 & 1.3 & 1 & 1.4 & 0.6 & 25 & 2.9 & 10 & 0.8 & $<0.0001$ \\
\hline Erythromycine & 205 & 15.2 & 4 & 6.6 & 4 & 5.7 & $<0.0001$ & 151 & 17.6 & 105 & 8.3 & $<0.0001$ \\
\hline TMP-SMXe & 227 & 16.9 & 92 & 13.0 & 9 & 12.9 & 0.06 & 150 & 17.5 & 178 & 14.0 & 0.03 \\
\hline Levofloxacin $^{\mathrm{e}}$ & 0 & 0 & 1 & 0.1 & 0 & 0 & 0.4 & 0 & 0 & 1 & 0.1 & 0.4 \\
\hline Total $^{f}$ & 1,347 & 100.0 & 710 & 100.0 & 70 & 100.0 & NA & 856 & 100.0 & 1,271 & 100.0 & NA \\
\hline
\end{tabular}

MIC: minimum inhibitory concentration; NA: not applicable.

Samples for which age group $(n=681)$ and/or geographical origin $(n=6)$ were not known are excluded from the respective analyses.

a $p$ value calculated with $3 \times 2$ chi-square test.

${ }^{b}$ Includes the French-speaking and bilingual French- and German-speaking Swiss Cantons.

'Includes the German- and Italian-speaking Cantons.

d $p$ value calculated with $2 \times 2$ chi-square test.

${ }^{e}$ Includes isolates with intermediate (i) and full antibiotic resistance (r).

${ }^{\mathrm{f}}$ Total number of isolates tested for antibiotic resistance. Susceptible isolates are not shown, but included in the total.

within individuals younger than five years and older than 64 years (Table 1 ).

Antibiotic resistance rates were highest in children younger than five years, for both invasive and noninvasive isolates, for all tested antibiotics (penicillin, ceftriaxone, erythromycin and TMP-SMX) with the exception of levofloxacin (Table 2). In general, antibiotic resistance rates were comparable between invasive and non-invasive isolates in all age groups. Non-susceptibility was higher in western Switzerland for penicillin, ceftriaxone, erythromycin and TMP-SMX for invasive as well as non-invasive isolates (Table 2).

Time trends of pneumococcal antibiotic resistance prevalence

We subsequently analysed the temporal evolution of antibiotic non-susceptibility rates for three different age groups (Table 3 and Figure 1). Chi-square test for trend over the individual years 2004 to 2014 showed significant decreasing trends for penicillin, erythromycin and TMP-SMX non-susceptibility in patients five to 64 years of age with invasive isolates. The same was true for the same three antibiotics in children younger than five years with non-invasive pneumococci. Further significant downward trends of non-susceptibility were seen in invasive isolates for erythromycin in the under five-year-olds and for ceftriaxone and TMP-SMX in the elderly (>64 years). The lowest non-susceptibility rates overall were found in the most recent years (2013-14), representing three and eight years, respectively, after the $\mathrm{PCV}_{13}$ and $\mathrm{PCV}_{7}$ vaccination recommendations for children under the age of two years.

\section{Serotype/serogroup-specific antibiotic} resistance was independent of the site of isolation

Figure 2 shows serotype/serogroup-specific antibiotic resistance for the three antibiotics penicillin, erythromycin and TMP-SMX. Results for ceftriaxone and levofloxacin were omitted as the numbers were low. The aORs were calculated for 22 serotypes/serogroups with individual proportions of above $1 \%$ overall (non-invasive and invasive isolates pooled). The serotypes consisting of serotypes/serogroups with overall individual proportions below $1 \%$ served as the reference group. Overall, ranking order of aORs of being non-susceptible revealed remarkable similarities between non-invasive 
Time trends of non-susceptibility of pneumococcal isolates by year ${ }^{\mathrm{a}}$, Switzerland, 2004-14 $(\mathrm{n}=13,125)$

\begin{tabular}{|c|c|c|c|c|c|c|c|c|c|c|c|c|c|c|}
\hline $\begin{array}{l}\text { Age } \\
\text { (years) }\end{array}$ & Non-susceptibility & 2004 & 2005 & 2006 & 2007 & 2008 & 2009 & 2010 & 2011 & 2012 & 2013 & 2014 & Total & Trend $^{b}$ \\
\hline \multicolumn{15}{|c|}{ Invasive S. pneumoniae } \\
\hline \multirow{4}{*}{$<5$} & Penicillin & 11 & 14 & 12 & 17 & 12 & 11 & 12 & 8 & 9 & 1 & 7 & 114 & 1 \\
\hline & Ceftriaxone & 2 & 7 & 3 & 6 & 4 & 4 & 2 & 1 & 3 & 0 & 1 & 33 & 0.2 \\
\hline & Erythromycin & 18 & 23 & 11 & 15 & 20 & 6 & 9 & 11 & 5 & 3 & 4 & 125 & 0.008 \\
\hline & TMP-SMX & 20 & 14 & 16 & 12 & 15 & 13 & 9 & 7 & 8 & 3 & 7 & 124 & 0.2 \\
\hline \multirow{4}{*}{$5-64$} & Penicillin & 43 & 48 & 28 & 31 & 42 & 46 & 45 & 28 & 37 & 25 & 19 & 392 & 0.009 \\
\hline & Ceftriaxone & 5 & 15 & 2 & 16 & 7 & 11 & 12 & 4 & 5 & 7 & 2 & 86 & 0.08 \\
\hline & Erythromycin & 46 & 39 & 41 & 49 & 46 & 66 & 40 & 29 & 37 & 29 & 18 & 440 & 0.0005 \\
\hline & TMP-SMX & 82 & 76 & 39 & 63 & 72 & 53 & 46 & 39 & 40 & 52 & 39 & 601 & $<0.0001$ \\
\hline \multirow{4}{*}{$>64$} & Penicillin & 29 & 43 & 48 & 48 & 45 & 47 & 68 & 47 & 46 & 34 & 28 & 483 & 0.4 \\
\hline & Ceftriaxone & 3 & 13 & 9 & 20 & 7 & 13 & 15 & 4 & 8 & 5 & 1 & 98 & 0.01 \\
\hline & Erythromycin & 44 & 50 & 65 & 84 & 76 & 61 & 53 & 56 & 57 & 51 & 49 & 646 & 0.1 \\
\hline & TMP-SMX & 80 & 88 & 53 & 69 & 68 & 68 & 64 & 48 & 68 & 44 & 43 & 693 & $<0.0001$ \\
\hline \multicolumn{2}{|l|}{ Total $^{\mathrm{c}}$} & 855 & 943 & 874 & 998 & 1,076 & 1,082 & 950 & 974 & 852 & 926 & 787 & & \\
\hline \multicolumn{15}{|c|}{ Non-invasive S. pneumoniae } \\
\hline \multirow{4}{*}{$<5$} & Penicillin & 44 & 46 & 44 & 24 & 15 & 13 & 18 & 16 & 6 & 3 & 1 & 230 & 0.006 \\
\hline & Ceftriaxone & 3 & 4 & 3 & 5 & 3 & 4 & 3 & 0 & 0 & 0 & 0 & 25 & 0.6 \\
\hline & Erythromycin & 38 & 43 & 38 & 24 & 12 & 10 & 13 & 13 & 8 & 3 & 3 & 205 & 0.01 \\
\hline & TMP-SMX & 52 & 50 & 33 & 24 & 14 & 7 & 15 & 14 & 6 & 7 & 5 & 227 & 0.002 \\
\hline \multirow{4}{*}{$5-64$} & Penicillin & 14 & 9 & 10 & 8 & 5 & 10 & 4 & 1 & 3 & 5 & 1 & 70 & 0.4 \\
\hline & Ceftriaxone & 2 & 0 & 2 & 2 & 2 & 1 & 0 & 0 & 0 & 0 & 0 & 9 & 0.3 \\
\hline & Erythromycin & 7 & 7 & 6 & 4 & 2 & 7 & 5 & 0 & 5 & 4 & 0 & 47 & 0.6 \\
\hline & TMP-SMX & 8 & 26 & 14 & 7 & 10 & 7 & 5 & 3 & 6 & 2 & 4 & 92 & 0.4 \\
\hline \multirow{4}{*}{$>64$} & Penicillin & 1 & 0 & 1 & 1 & 0 & 0 & 0 & 0 & 0 & 0 & 0 & 3 & 0.08 \\
\hline & Ceftriaxone & 0 & 0 & 1 & 0 & 0 & 0 & 0 & 0 & 0 & 0 & 0 & 1 & 0.4 \\
\hline & Erythromycin & 0 & 0 & 1 & 0 & 1 & 0 & 0 & 1 & 1 & 0 & 0 & 4 & 0.8 \\
\hline & TMP-SMX & 2 & 1 & 1 & 1 & 1 & 1 & 0 & 0 & 1 & 0 & 1 & 9 & 0.2 \\
\hline \multicolumn{2}{|l|}{ Total $^{\mathrm{c}}$} & 340 & 344 & 354 & 193 & 181 & 182 & 137 & 147 & 118 & 70 & 61 & & \\
\hline
\end{tabular}

NA: not applicable; TMP-SMX: trimethoprim/sulfamethoxazole.

Samples for which age group was not known $(n=681)$ are excluded from this analysis.

${ }^{a}$ White shading: pre-PCV7 years; light grey shading: post-PCV7 but pre-PCV13 years; dark grey shading: post-PCV13 years.

${ }^{b} \mathrm{p}$ value calculated by chi-square test for trend. Significant $\mathrm{p}$ values indicated in bold (i.e. $p<0.05$ ).

c Total number of isolates tested in all three age groups combined for which patient age was known (total invasive isolates: $n=10,317$; total non-invasive isolates: $n=2,127)$. Susceptible isolates are not shown, but included in the total.

and invasive S. pneumoinae isolates. The four serotypes with lowest susceptibility and therefore with the highest aORs for penicillin were identical in the groups of non-invasive and invasive isolates (19A, 19F, 14 and 15; Figures $2 \mathrm{~A}$ and $\mathrm{B}$ ). For TMP-SMX, the order of aORs was different between invasive and non-invasive isolates, but the serotype with the lowest susceptibility (serotype 9V) was again identical (Figures $2 \mathrm{C}$ and D). Finally, serogroups 14 and 33 had the highest aORs for erythromycin non-susceptibility in both collections (Figures 2E and F). Thus, a distinct, serotype-specific antibiotic resistance exists and is likely to be independent of the anatomical isolation site (Figures 2A-2F).

Most serotypes revealing significantly higher antibiotic resistance rates than the reference group were covered by $\mathrm{PCV}_{13}$ (Figures $2 \mathrm{~A}-2 \mathrm{~F}$ ), and indeed, introduction of the vaccine mitigated the prevalence of pneumococcal antibiotic resistance (Table 3). However, non-PCV13 serogroups/serotypes $15,6 \mathrm{C}$ and 33 were also associated with increased antibiotic resistance to penicillin and/or erythromycin.

\section{Serotype/serogroup distribution by geographical region}

We calculated the serotype/serogroup-specific antibiotic resistance of the four most resistant $S$. pneumoniae serotypes for the three antibiotics penicillin, TMP-SMX and erythromycin by geographical region in Switzerland (west vs other; Table 4). We found that the geographical region had a profound effect on serotype/serogroup-specific antibiotic resistance (Table 4). In order to determine if these differences were significant, we performed a logistic regression for each 
TABLE 4

Serotype/serogroup-specific penicillin resistance of invasive and colonising Streptococcus pneumoniae isolates, by geographical region, Switzerland, 2004-14 $(\mathrm{n}=13,125)$

\begin{tabular}{|c|c|c|c|c|c|c|}
\hline \multirow{2}{*}{$\begin{array}{l}\text { Serotype/ } \\
\text { serogoup }\end{array}$} & \multirow{2}{*}{ Number of isolates ${ }^{b}$} & \multirow{2}{*}{ Antibiotic } & \multicolumn{3}{|c|}{$\%$ SSR } & \multirow{2}{*}{ Adjusted OR $(95 \% \mathrm{Cl})$ for West ${ }^{\mathrm{a}}$} \\
\hline & & & All & West & Other & \\
\hline $19 \mathrm{~A}$ & 884 & \multirow{4}{*}{ Penicillin } & 32.4 & 44.9 & 25.9 & $2.2(1.6-3.1)$ \\
\hline $19 \mathrm{~F}$ & 663 & & 30.3 & 46.5 & 22.7 & $3.0(2.0-4.4)$ \\
\hline 14 & 1,095 & & $24 \cdot 7$ & 50.8 & 16.2 & $5.3(3.8-7.4)$ \\
\hline 15 & 338 & & 23.7 & 26.9 & 21.9 & $1.6(0.9-2.9)$ \\
\hline $9 \mathrm{~V}$ & 526 & \multirow{4}{*}{ TMP-SMX } & $44 \cdot 9$ & 58.0 & 40.2 & $2.1(1.4-3.3)$ \\
\hline $23 \mathrm{~F}$ & 632 & & 29.1 & 34.8 & 26.8 & $1.6(1.1-2.5)$ \\
\hline $6 \mathrm{~B}$ & 374 & & 27.8 & 28.6 & 27.5 & $1.0(0.6-1.8)$ \\
\hline $18 \mathrm{C}$ & 304 & & 27.6 & 30.1 & 26.7 & $1.2(0.6-2.2)$ \\
\hline 14 & 1,095 & \multirow{4}{*}{ Erythromycin } & $49 \cdot 3$ & 61.9 & 45.2 & $1.8(1.4-2.5)$ \\
\hline 33 & 160 & & 33.1 & $54 \cdot 4$ & 24.6 & $3.7(1.5-9.5)$ \\
\hline $19 \mathrm{~A}$ & 884 & & 24.9 & 33.2 & 20.6 & $2.0(1.4-2.8)$ \\
\hline 15 & 338 & & 24.0 & 31.9 & 19.6 & $2.4(1.4-4.3)$ \\
\hline
\end{tabular}

$\mathrm{Cl}$ : confidence interval; OR: odds ratio; SSR: serotype-specific resistance; TMP-SMX: trimethoprim/sulfamethoxazole.

a ORs of antibiotic-resistant serotypes were adjusted for year of isolation, age category and site of isolation (invasive versus non-invasive). Isolates from the 'other' part of Switzerland were used as the reference group.

b Isolates with unknown age $(n=681)$ and/or geographical region known $(n=6)$ were excluded. Only results for serotypes/serogroups with high SSR are shown (i.e. 19A, 19F, 14, 15, 9V, 23F, 6B, 18C and 33).

serotype/serogroup, using the isolates from the other part of Switzerland as the reference group.

For penicillin, erythromycin and TMP-SMX, three of four $(14,19 \mathrm{~F}$ and $19 \mathrm{~A})$, four of four $(33,15,19 \mathrm{~A}$ and 14$)$ and two of four serotypes/serogroups (9V, 23F) showed significantly higher rates in the west than in the rest of the country. This may partly explain the higher nonsusceptibility rates for all antibiotics found in the west compared with the rest of the country (Table 2 ).

\section{Discussion}

Our study concurrently compared antibiotic resistance rates from the non-invasive and invasive pneumococcal surveillance systems in Switzerland from 2004 to 2014. Overall, serotype/serogroup and antibiotic resistance data was available for more than 13,000 non-invasive or invasive isolates. The main findings of this study were (i) that antibiotic resistance of pneumococci decreased after the vaccination recommendation of $\mathrm{PCV}_{7}$ and PCV13 and (ii) that patient age and geographical origin of samples had a greater influence on the epidemiology of antibiotic resistance of pneumococci than the site of isolation (i.e. invasive or non-invasive).

Compared with other European countries, the antibiotic resistance rates of the isolates were generally lower in our setting [11]. The French pneumococcal surveillance network analysed the antibiotic susceptibility of 6,683 S. pneumoniae isolated from children with acute otitis media from 2001 to 2011 [12]. Non-susceptibility (intermediate and full resistance) rates in 2011 were $57.3 \%$ for penicillin in these French isolates. It is generally difficult to compare absolute penicillin resistance data from different studies from different countries because the guidelines for the interpretation of antibiotic resistance have changed (e.g. to apply CLSI versus European Committee on Antimicrobial Susceptibility Testing (EUCAST) breakpoints) However, we identified lower penicillin non-susceptibility rates of $14 \%$ within our non-invasive isolates in our study compared with the French study. One possible explanation for these discrepancies between two neighbouring countries might be outpatient antibiotic use which is very low in Switzerland compared with other European countries such as France [13].

Furthermore, we revealed that antibiotic resistance decreased over time in patients up to age 64 years from 2004 to 2014 . This can be attributed to the decrease of non-susceptible serotypes such as $19 \mathrm{~A}, 9 \mathrm{~V}, 6 \mathrm{~B}, 23 \mathrm{~F}$ and 14 among invasive and non-invasive $S$. pneumoniae, which is most likely due to $\mathrm{PCV}_{7}$ and $P C V_{13}$ vaccination as seen in other studies [12,14-18]. However, it has to be noted that in patients older than 64 years with invasive $S$. pneumoniae, with the exception of TMP-SMX and ceftriaxone, resistance rates remained unchanged from 2004 to 2014, although a serotype redistribution took place also in this age group $[4,19]$. In the future, serotypes $6 \mathrm{C}$ and serogroups 15 and 35 should be followed up carefully as they are the most prominent non-PCV13 serotypes associated with antibiotic resistance. Serotype 6C and serogroup 15 have been implicated in other recent studies as emerging, resistant serotypes $[20,21]$.

Whether invasive and non-invasive pneumococcal isolates inherently differ in resistance rates is still unclear $[22,23]$. In our study, differences in the ranking of ORs for penicillin, TMP-SMX and erythromycin between 
non-invasive and invasive $S$. pneumoniae were minimal, after correcting for age, time and geographical region. Indeed, the rankings of the ORs of the more resistant serotypes for each of the three antibiotics were identical for invasive and non-invasive $S$. pneumoniae. However, the specific setting in which noninvasive isolates were received may be crucial. While we used isolates collected in primary care in situations that do not normally warrant a microbiological diagnosis (uncomplicated otitis media and communityacquired pneumonia), many other studies used data from non-invasive pneumococci generated in routine microbiological work-up $[7,24,25]$. It is well known that using routine microbiological data may select for more complicated and more often pre-treated cases, which in turn increases the chance of finding antibiotic-resistant isolates $[26,27]$. We therefore think that the design of our network may reflect a more general situation of antibiotic resistance for these isolates.

Besides temporal trends, we also analysed geographical differences in resistance rates. We found that resistance rates were higher in the west than in the rest of Switzerland. We saw only minor dissimilarities in overall serotype distribution, which cannot explain these geographical differences (data not shown). Therefore, an explanation for these discrepancies could be varying antibiotic consumption. Indeed, higher overall antibiotic consumption has been observed in western Switzerland in in- [28] and outpatients [13,29]. More specifically, a higher antibiotic consumption in the west was found for beta-lactams and macrolides but not for TMP-SMX. This is in line with the higher serotype/serogroup-specific resistance against penicillin and erythromycin but not against TMP-SMX that we observed in western Switzerland. A clear correlation between antibiotic resistance and outpatient use of penicillin has been described previously [11].

This study has some major strengths. It includes a large data volume, allowing for demographic and temporal stratification and the parallel analysis of data from invasive and non-invasive isolates with a great statistical power. Furthermore, it includes congruent analysis of invasive and non-invasive samples in the same laboratory with the same methodology and constantly over a long observation time of 11 years, spanning the introduction of $P C V_{7}$ and $P C V_{13}$ vaccines. The non-invasive samples were true outpatient samples and were collected by ca 200 practitioners, representative of Swiss primary care physicians.

There are some limitations to this study. Due to the characteristics of the collection of S. pneumoniae isolates, patients with invasive $S$. pneumoniae differed significantly in age and regional origin from those with colonising S. pneumoniae. However, the large number of included isolates still allowed for subgroup analysis and adjusting for potential confounding factors in logistic regression models. In addition, colonising $S$. pneumoniae were collected from patients with pneumococcal disease (acute otitis media or pneumonia) but not from those with other diseases such as acute exacerbation of chronic obstructive pulmonary disease (AECOPD) [5,16] or from healthy people. Not including patients with AECOPD may reduce observed rates of antibiotic non-susceptibility as these patients are more likely to have received antibiotics (as discussed above) [26].

\section{Conclusion}

In summary, this is a detailed and comprehensive study analysing antibiotic resistance rates and serotype distribution with high statistical power in invasive and noninvasive S. pneumoniae in Switzerland. We revealed that antibiotic non-susceptibility was highest in children under five years of age. Furthermore, we showed that variations in the regional antibiotic consumption and the introduction of vaccines may have influenced antibiotic non-susceptibility rates. The introduction of $\mathrm{PCV}_{7}$ and $\mathrm{PCV}_{13}$ markedly reduced resistance rates in pneumococci in Switzerland, mainly by decreasing the prevalence of the most resistant serotypes/serogroups. Site of isolation (invasive vs non-invasive) by itself had a much more limited influence on the epidemiology. At the end of the study period in 2014, distinct antibiotic resistance rates were on an all-time low for various age groups, but certain non-PCV13 resistant serotypes have to be carefully monitored in the future. The two described national surveillance systems are both important to guide future antibiotic stewardship and vaccination policies in Switzerland.

\section{Acknowledgements}

We thank Marianne Küffer and Chantal Studer for their help with the serotyping. We also acknowledge the long standing support of the Federal Office of Public Health (FOPH). We also owe many thanks to all the laboratories of Clinical Microbiology in Switzerland for the excellent partnership within this national surveillance system. We are very grateful to all the physicians who enthusiastically take part in the Sentinella surveillance system and who provided non-invasive sample material.

The Swiss National Reference Centre for Pneumococci (NZPn) in Switzerland and the prospective surveillance study on colonising pneumococci within Sentinella are funded by the $\mathrm{FOPH}$.

\section{Conflict of interest}

An educational grant from Pfizer AG for partial support of this project was received. However, the sponsor had no role in the data analysis and content of the manuscript.

\section{Authors' contributions}

All authors conceived and designed the study; $\mathrm{CH}, \mathrm{AK}, \mathrm{MH}$ analysed the data and wrote the manuscript. $\mathrm{CH}, \mathrm{AK}, \mathrm{AA}, \mathrm{MH}$ contributed to the discussion and reviewed the manuscript. $\mathrm{CH}, \mathrm{AK}, \mathrm{AA}, \mathrm{MH}$ saw, commented upon and approved the final version of the paper. 


\section{References}

1. Aebi C, Duppenthaler A. [Antimicrobial resistance-consequences for ambulatory treatment of infections in children]. Ther Umsch. 2002;59(1):46-50. German. DOI: 10.1024/0040-5930.59.1.46 PMID: 11851047

2. Feikin DR, Schuchat A, Kolczak M, Barrett NL, Harrison LH, Lefkowitz L, et al. Mortality from invasive pneumococcal pneumonia in the era of antibiotic resistance, 1995-1997. Am Public Health. 2000;90(2):223-9. DOI: 10.2105/AJPH.90.2.223 PMID: 10667183

3. Schwaber MJ, De-Medina T, Carmeli Y. Epidemiological interpretation of antibiotic resistance studies - what are we missing?Nat Rev Microbiol. 2004;2(12):979-83. DOI: 10.1038/ nrmicr01047 PMID: 15550943

4. Meichtry J, Born R, Küffer M, Zwahlen M, Albrich WC, Brugger $\mathrm{SD}$, et al. Serotype epidemiology of invasive pneumococcal disease in Swiss adults: a nationwide population-based study. Vaccine. 2014:32(40):5185-91. DOI: 10.1016/j. vaccine.2014.07.060 PMID: 25077419

5. Song JH, Dagan R, Klugman KP, Fritzell B. The relationship between pneumococcal serotypes and antibiotic resistance.Vaccine. 2012;30(17):2728-37. DOI: 10.1016/j. vaccine.2012.01.091 PMID: 22330126

6. Kronenberg A, Zucs P, Droz S, Mühlemann K. Distribution and invasiveness of Streptococcus pneumoniae serotypes in Switzerland, a country with low antibiotic selection pressure, from 2001 to 2004.J Clin Microbiol. 2006;44(6):2032-8. DOI: 10.1128/JCM.00275-06 PMID: 16757594

7. Hoban DJ, Doern GV, Fluit AC, Roussel-Delvallez M, Jones RN. Worldwide prevalence of antimicrobial resistance in Streptococcus pneumoniae, Haemophilus influenzae, and Moraxella catarrhalis in the SENTRY Antimicrobial Surveillance Program, 1997-1999.Clin Infect Dis. 2001;32(Suppl 2):S81-93.

8. Mühlemann K, Matter HC, Täuber MG, Bodmer T, Sentinel Working Group. Nationwide surveillance of nasopharyngeal Streptococcus pneumoniae isolates from children with respiratory infection, Switzerland, 1998-1999.J Infect Dis. 2003;187(4):589-96. DOI: 10.1086/367994 PMID: 12599075

9. Bundesamt für Gesundheit (BAG). Repräsentativität des Sentinella-Ärztekollektivs. [Representativeness of the collective of sentinel physicians]. BAG Bulletin. 2001;37(01):680. German. Available from: http://www.bag. admin.ch/dokumentation/publikationen/01435/01801/index.ht $\mathrm{ml}$ ?lang=de\&download=NHzLpZig $7 \mathrm{t}$, Inp 6loNTU0 42 l 2 Z 6 In 1 acy 4Zn4Z2qZpnO2Yuq2Z6gpJCEdX 56 fWym162dpYbUzd,Gpd6emK2 OzgaGodetmqaN19XI2IdvoaCUZ,s

10. Clinical and Laboratory Standards Institute (CLSI). Performance standards for antimicrobial susceptibility testing, twentysecond informational supplement. Document M100-S22. Wayne: CLSI; 2012

11. Goossens H, Ferech M, Vander Stichele R, Elseviers M, ESAC Project Group. Outpatient antibiotic use in Europe and association with resistance: a cross-national database study.Lancet. 2005;365(9459):579-87. DOI: 10.1016/S01406736(05)70799-6 PMID: 15708101

12. Kempf M, Varon E, Lepoutre A, Gravet A, Baraduc R, Brun $M$, et al. Decline in antibiotic resistance and changes in the serotype distribution of Streptococcus pneumoniae isolates from children with acute otitis media; a 2001-2011 survey by the French Pneumococcal Network. Clin Microbiol Infect. 2015;21(1):35-42. DOI: 10.1016/j.cmi.2014.08.009 PMID: 25636925

13. Filippini M, Masiero G, Moschetti K. Socioeconomic determinants of regional differences in outpatient antibiotic consumption: evidence from Switzerland. Health Policy. 2006;78(1):77-92. DOI: 10.1016/j.healthpol.2005.09.009 PMID: 16290129

14. Imöhl M, Reinert RR, Mutscher C, van der Linden M. Macrolide susceptibility and serotype specific macrolide resistance of invasive isolates of Streptococcus pneumoniae in Germany from 1992 to 2008.BMC Microbiol. 2010;10(1):299. DOI: 10.1186/1471-2180-10-299 PMID: 21108778

15. Kyaw MH, Lynfield R, Schaffner W, Craig AS, Hadler J, Reingold $A$, et al. Effect of introduction of the pneumococcal conjugate vaccine on drug-resistant Streptococcus pneumoniae. N Engl J Med. 2006;354(14):1455-63. DOI: 10.1056/NEJMoa051642 PMID: 16598044

16. Hackel M Lascols C, Bouchillon S, Hilton B, Morgenstern D, Purdy J. Serotype prevalence and antibiotic resistance in Streptococcus pneumoniae clinical isolates among global populations.Vaccine. 2013;31(42):4881-7. DOI: 10.1016/j. vaccine.2013.07.054 PMID: 23928466

17. Imöhl M, Reinert RR, van der Linden M. Serotype-specific penicillin resistance of Streptococcus pneumoniae in Germany from 1992 to 2008.Int J Med Microbiol. 2010;300(5):324-30. DOI: 10.1016/j.ijmm.2009.11.004 PMID: 20071233
18. Dagan R, Juergens C, Trammel J, Patterson S, Greenberg $D$, Givon-Lavi N, et al. Efficacy of 13-valent pneumococcal conjugate vaccine (PCV13) versus that of 7-valent PCV $\left(\mathrm{PCV}_{7}\right)$ against nasopharyngeal colonization of antibioticnonsusceptible Streptococcus pneumoniae. J Infect Dis. 2015;211(7):1144-53. DOI: 10.1093/infdis/jiu576 PMID: 25355940

19. Bundesamt für Gesundheit (BAG). PneumokokkenErkrankungen. [Pneumococcal diseases]. Bern: BAG; 2013. German. Available from: http://www.bag.admin.ch/themen/ medizin/00682/00684/01097/index.html?lang=it

20. Chiba N, Morozumi M, Shouji M, Wajima T, Iwata S, Ubukata K, et al. Changes in capsule and drug resistance of Pneumococci after introduction of PCV7, Japan, 2010-2013. Emerg Infect Dis. 2014;20(7):1132-9. DOI: 10.3201/eid2007.131485 PMID: 24960150

21. Regev-Yochay G, Paran Y, Bishara J, Oren I, Chowers M, Tziba $Y$, et al. Early impact of PCV $7 / P^{2} V_{13}$ sequential introduction to the national pediatric immunization plan, on adult invasive pneumococcal disease: A nationwide surveillance study. Vaccine. 2015;33(9):1135-42. DOI: 10.1016/j. vaccine.2015.01.030 PMID: 25613717

22. Lambertsen LM, Harboe ZB, Konradsen HB, Christensen J, Hammerum AM. Non-invasive erythromycin-resistant pneumococcal isolates are more often non-susceptible to more antimicrobial agents than invasive isolates. Int J Antimicrob Agents. 2010;35(1):72-5. DOI: 10.1016/j. ijantimicag.2009.09.006 PMID: 19892529

23. Saha SK, Baqui AH, Darmstadt GL, Ruhulamin M, Hanif M, El Arifeen S, et al. Comparison of antibiotic resistance and serotype composition of carriage and invasive pneumococci among Bangladeshi children: implications for treatment policy and vaccine formulation. J Clin Microbiol. 2003;41(12):5582-7. DOI: $10.1128 / J C M .41 .12 .5582-5587.2003$ PMID: 14662944

24. Jacobs MR, Felmingham D, Appelbaum PC, Grüneberg RN, Alexander Project Group. The Alexander Project 1998-2000: susceptibility of pathogens isolated from community-acquired respiratory tract infection to commonly used antimicrobia agents.J Antimicrob Chemother. 2003;52(2):229-46. DOI: 10.1093/jac/dkg321 PMID: 12865398

25. Beekmann SE, Heilmann KP, Richter SS, García-de-Lomas J, Doern GV, GRASP Study Group. Antimicrobial resistance in Streptococcus pneumoniae, Haemophilus influenzae, Moraxella catarrhalis and group A beta-haemolytic streptococci in 2002-2003. Results of the multinational GRASP Surveillance Program.Int J Antimicrob Agents. 2005;25(2):14856. DOI: 10.1016/j.ijantimicag.2004.09.016 PMID: 15664485

26. Pérez-Trallero E, Marimón JM, Larruskain J, Alonso M, Ercibengoa M. Antimicrobial susceptibilities and serotypes of Streptococcus pneumoniae isolates from elderly patients with pneumonia and acute exacerbation of chronic obstructive pulmonary disease.Antimicrob Agents Chemother. 2011;55(6):2729-34. DOI: 10.1128/AAC.01546-10 PMID: 21402843

27. Kronenberg A, Koenig S, Droz S, Mühlemann K. Active surveillance of antibiotic resistance prevalence in urinary tract and skin infections in the outpatient setting.Clin Microbiol Infect. 2011;17(12):1845-51. DOI: 10.1111/j.14690691.2011.03519.x PMID: 21880098

28. Plüss-Suard C, Pannatier A, Kronenberg A, Mühlemann K, Zanetti G. Hospital antibiotic consumption in Switzerland: comparison of a multicultural country with Europe.J Hosp Infect. 2011;79(2):166-71. DOI: 10.1016/j.jhin.2011.05.028 PMID: 21820207

29. Achermann R, Suter K, Kronenberg A, Gyger P, Mühlemann $\mathrm{K}$, Zimmerli W, et al. Antibiotic use in adult outpatients in Switzerland in relation to regions, seasonality and point of care tests. Clin Microbiol Infect. 2011;17(6):855-61. DOI: 10.1111/j.1469-0691.2010.03348.x PMID: 20731682

\section{License and copyright}

This is an open-access article distributed under the terms of the Creative Commons Attribution (CC BY 4.0) Licence. You may share and adapt the material, but must give appropriate credit to the source, provide a link to the licence, and indicate if changes were made.

This article is copyright of the authors, 2016. 